\title{
Deconfinement and color screening in 2+1 flavor QCD
}

\author{
Péter Petreczky ${ }^{1, a}$ and Johannes Weber ${ }^{2, b}$ \\ ${ }^{1}$ Brookhaven National Laboratory, Physics Department, Upton NY 11973-5000, USA \\ ${ }^{2}$ Technische Universität München, Physik Department T30f, James-Franck-Str. 1, 85748 Garching, Ger- \\ many
}

\begin{abstract}
We discuss the deconfinement an color screening in $2+1$ flavor QCD in terms of the free energy of static quarks.
\end{abstract}

\section{Introduction}

QCD is known to exhibit a confined phase at low temperatures and a deconfined phase at high temperatures. In the confined phase, quarks and gluons are bound inside of hadrons and the iso-vector chiral symmetry in the light quark sector is broken spontaneously. This spontaneously broken chiral symmetry is restored in the deconfined phase.

In $S U\left(N_{c}\right)$ pure gauge theory without dynamical quarks, the deconfinement transition is a phase transition, with the Polyakov loop being the order parameter. This phase transition is associated with the $Z\left(N_{c}\right)$ center symmetry. The logarithm of the Polyakov loop corresponds to the negative free energy of a static quark in temperature units [1]. The Polyakov loop is not invariant under $Z\left(N_{c}\right)$ transformation and therefore strictly vanishes for temperatures below the phase transition temperature, i.e. the free energy of a static quark is infinite. On the other hand, it assumes a finite expectation value above the transition temperature due to color screening. Thus, the deconfinement transition is closely related to the onset of color screening [1].

The center symmetry is broken explicitly by the presence of sea quarks in full QCD. As two color charges are separated beyond the string breaking distance, the flux tube breaks apart and a light quark-antiquark pair is created from the sea. Hence, the Polyakov loop takes a small, but positive expectation value in the confined phase of QCD, which is related to the binding energy of static-light mesons. Therefore, it is not clear to what extent the Polyakov loop is suitable for the discussion of the deconfinement transition in QCD, and therefore, the deconfinement in QCD is often discussed in terms of fluctuations of conserved charges (see [2] and references therein).

In this contribution we discuss the deconfinement transition and color screening in QCD in terms of the Polyakov loop or equivalently the free energy $F_{Q}(T)$ of a static quark. We will also consider related quantities like the singlet free energy $F_{1}(r, T)$ and the color-averaged free energy $F_{\text {avg }}(r, T)$ of a static quark anti-quark pair in full QCD.

\footnotetext{
a e-mail: petreczk@quark.phy.bnl.gov

be-mail: johannes.weber@tum.de
} 


\section{Lattice setup}

We study the Polyakov loop and related correlators in full QCD for a large range of temperatures and four different temporal extents $N_{\tau}=\{12,10,8,6\}$ of the lattice. The temperature $T$ is related to the gauge coupling $\beta$ through the lattice spacing $a(\beta)$ and is defined as $T\left(\beta, N_{\tau}\right)=1 /\left(a(\beta) N_{\tau}\right)$. Taking the continuum limit at fixed temperature requires simultaneous variation of both $\beta$ and $N_{\tau}$, which is why numerical studies of QCD at finite temperature are quite demanding. In order to avoid finite volume effects, we use lattices with aspect ratio $N_{\sigma} / N_{\tau}=4$, where $N_{\sigma}$ is the extent of each spatial direction. We have physical strange quark mass and nearly physical light quark masses, $m_{l}=m_{s} / 20$, corresponding to a pion mass of $m_{\pi}=161 \mathrm{MeV}$ in the continuum limit. The gauge configurations were generated with tree-level improved Symanzik gauge action and highly improved staggered quarks (HISQ) by HotQCD collaboration [8, 9]. We use the publicly available MILC code [3] in combination with libraries provided by the USCQD consortium [4].

\subsection{Observables}

The Polyakov loop $L\left(\beta, N_{\tau}, \mathbf{x}\right)$ is defined in lattice QCD as a normalized trace of a temporal Wilson line $W\left(\beta, N_{\tau}, \mathbf{x}\right)$ wrapping around the time direction once,

$$
L\left(\beta, N_{\tau}, \mathbf{x}\right)=\frac{1}{N_{c}} \operatorname{Tr} W\left(\beta, N_{\tau}, \mathbf{x}\right), \quad W\left(\beta, N_{\tau}, \mathbf{x}\right)=\prod_{x_{0}=1}^{N_{\tau}} U_{0}\left(x_{0}, \mathbf{x}\right) .
$$

We obtain the expectation values of Polyakov loop, Polyakov loop correlator and color-singlet Wilson line correlator by taking the ensemble average (average over $\mathbf{x}$ included) as

$$
\begin{aligned}
L^{\text {bare }}\left(\beta, N_{\tau}\right) & =\left\langle L\left(\beta, N_{\tau}, \mathbf{x}\right)\right\rangle \\
C_{P}^{\text {bare }}\left(\beta, N_{\tau}, r\right) & =\left\langle L\left(\beta, N_{\tau}, \mathbf{x}\right) L^{\dagger}\left(\beta, N_{\tau}, \mathbf{x}+\mathbf{r}\right)\right\rangle \\
C_{S}^{\text {bare }}\left(\beta, N_{\tau}, r\right) & =\left\langle\frac{1}{N_{c}} \operatorname{Tr}\left[W\left(\beta, N_{\tau}, \mathbf{x}\right) W^{\dagger}\left(\beta, N_{\tau}, \mathbf{x}+\mathbf{r}\right)\right]\right\rangle .
\end{aligned}
$$

Since the Wilson line $W\left(\beta, N_{\tau}, \mathbf{x}\right)$ itself is not gauge invariant, $C_{S}^{\text {bare }}\left(\beta, N_{\tau}, r\right)$ vanishes unless a gauge-fixing procedure is used. We fix the gauge fields to Coulomb gauge $[5,6]$. All three bare observables diverge in the continuum limit and require multiplicative renormalization with a factor $\exp \left[-c_{Q}(\beta)\right]$ for each matrix $W(\mathbf{x})$. The renormalized observables are related to free energies for finite $N_{\tau}$ and in the continuum limit as

$$
L^{\mathrm{ren}}(T)=e^{-F_{Q}(T) / T} \quad, \quad C_{P}^{\mathrm{ren}}(T, r)=e^{-F_{\mathrm{avg}}(r, T) / T} \quad \text { and } \quad C_{S}^{\mathrm{ren}}(T, r)=e^{-F_{S}(r, T) / T} .
$$

In the following discussion, we make use of free energies in units of the temperature, e.g. $f_{Q}\left(T, N_{\tau}\right)=F_{Q}\left(T, N_{\tau}\right) / T$, which vary more slowly with the temperature than the primary observables and are renormalized by adding $N_{\tau} c_{Q}(\beta)$ (or the respective combinations for the other free energies). We also usually omit the indication ${ }^{\text {ren }}$ for renormalized quantities.

\subsection{Renormalization and scale setting with $Q \bar{Q}$ procedure}

We obtain the renormalization constant $c_{Q}(\beta)$ by normalizing the static $Q \bar{Q}$ potential at zero temperature $V(r)$ to a prescribed value. Namely, the static potential at zero temperature 
for different lattice spacings $a$ is fixed to be $0.954 / r_{0}$ or $0.2065 / r_{1}$ at distances $r=r_{0}$ [8] or $r=r_{1}[9]$, respectively. The scales $r_{0}$ and $r_{1}$ are defined as

$$
\left.r^{2} \frac{d V(r)}{d r}\right|_{r=r_{i}}=C_{i}, \quad i=0,1,
$$

where $C_{0}=1.65$ and $C_{1}=1.0$. In physical units we have $r_{0}=0.4688(41) \mathrm{fm}$ and $r_{1}=$ $0.3106 \mathrm{fm}$ [7]. Either distance $r_{i}$ defines the lattice spacing $a(\beta)$ in physical units as a function of the gauge coupling $\beta=10 / g^{2}$. The renormalization procedure ( $Q \bar{Q}$ procedure) is limited by availability of lattice data at zero temperature $(\beta \leq 7.825$, lattice spacing $a \gtrsim 0.04 \mathrm{fm})$. Thus, continuum extrapolation including the finest lattices $\left(N_{\tau}=12\right.$ is restricted to $T \lesssim 407 \mathrm{MeV}$. The renormalization constants $c_{Q}(\beta)$ have been obtained using the results on $V(r)$ from $[8,9]$. We have to interpolate the renormalization constant in $\beta$, since we require intermediate $\beta$ values for renormalization at fixed temperature. We interpolate $c_{Q}(\beta)$ using $\mathrm{R}$ statistical package [10] with error propagation via bootstrap method. Further details of the interpolation are deferred to a publication in preparation [12].

\section{Deconfinement observables from the Polyakov loop}

\subsection{Free energy $F_{Q}(T)$}

We use bare Polyakov loop data from HotQCD given in $[8,9]$ and interpolate $f_{Q}^{\text {bare }}\left(\beta, N_{\tau}\right)$ for each $N_{\tau}$ in $\beta$ using $\mathrm{R}$ statistical package [10] with error propagation via bootstrap method. Further details of the interpolation are deferred to a publication in preparation [12]. Interpolation is necessary to obtain the Polyakov loop for a set of common temperatures for all $N_{\tau}$. We add the errors of $N_{\tau} c_{Q}(\beta)$ in quadrature, since they are statistically independent from the errors of the bare Polyakov loop.

Above $T \gtrsim 200 \mathrm{MeV}$, cutoff effects are similar to the size of uncertainties of the interpolation, which are dominated by the uncertainty of $N_{\tau} c_{Q}(\beta)$. We extrapolate $F_{Q}\left(T, N_{\tau}\right)$ to the continuum limit with point-wise extrapolations for each temperature and with a global fit using a polynomial Ansatz,

$$
F_{Q}\left(T, N_{\tau}\right)=\sum_{i_{0}=0}^{N_{0}} a_{i_{0}} T^{i_{0}}+\frac{1}{N_{\tau}^{2}} \sum_{i_{2}=0}^{N_{2}} a_{i_{2}} T^{i_{2}} .
$$

The Ansatz is specified in terms of the orders $N_{0}$ and $N_{2}$ of two temperature polynomials, which parametrize the temperature dependence of the continuum limit and of cutoff effects. In both cases, the extrapolations are performed with and without the $N_{\tau}=6$ data. We finally include estimates of the uncertainties of the continuum extrapolation using different global and point-wise fits. Further details of the interpolation are deferred to a publication in preparation [12].

Our continuum result is shown as a black band in figure 1. This result has been discussed already in a comparison between conventional approach to the renormalization of the Polyakov loop and the renormalization of the Polyakov with gradient flow [11]. It has been found that the continuum limit of $F_{Q}\left(T, N_{\tau}\right)$ at finite flow time is consistent up to an additive constant with $F_{Q}(T)$ using $Q \bar{Q}$ procedure for temperatures up to $T \lesssim 400 \mathrm{MeV}$. We show comparison of results for $F_{Q}(T, 12)$ using either $Q \bar{Q}$ procedure or flow time $f_{t}=f_{0}$ in figure 1. Details of the gradient flow scheme and the choice for $f_{0}$ are discussed in [11]. Both renormalization 


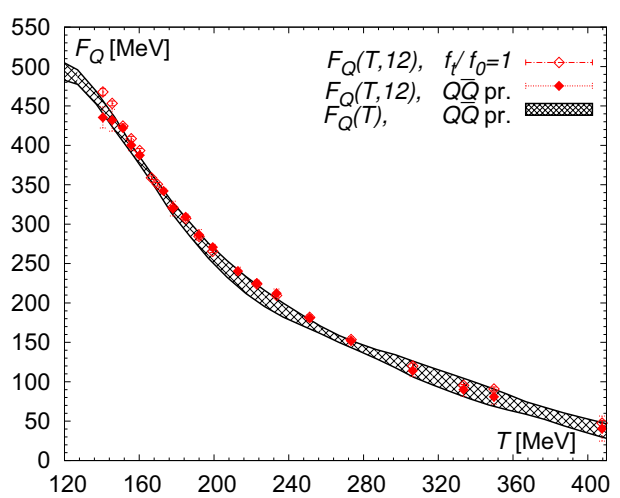

Figure 1. Continuum limit $F_{Q}(T)$ using $Q \bar{Q}$ procedure as black $1 \sigma$ band. Error bars show $F_{Q}(T, 12)$ in $Q \bar{Q}$ procedure (filled symbols) and at flow time $f_{t}=f_{0}$ (open symbols). The flow time result is taken from [11].

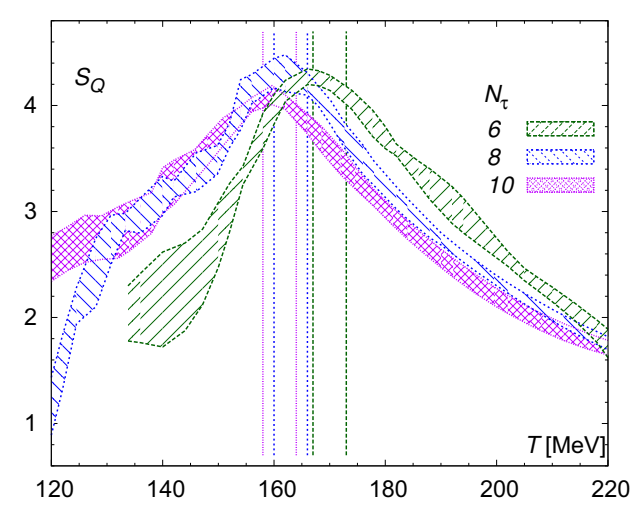

Figure 2. $S_{Q}\left(T, N_{\tau}\right)$ using $Q \bar{Q}$ procedure has a peak close to $T_{c}\left(N_{\tau}\right)$, which is indicated by the three pairs of vertical lines.

schemes agree within errors for $N_{\tau}=12$ with exception of a few low temperature points which have relatively poor statistics. For coarser lattices, flow-time dependent cutoff effects are observed for low temperatures, but are taken care of by continuum extrapolation.

\subsection{Entropy $S_{Q}(T)$}

The temperature derivative of the free energy is related to the entropy of a static quark as

$$
S_{Q}\left(T, N_{\tau}\right)=-\frac{\partial F_{Q}\left(T, N_{\tau}\right)}{\partial T}=\log L\left(T, N_{\tau}\right)+\frac{L^{\prime}\left(T, N_{\tau}\right)}{L\left(T, N_{\tau}\right)}, \quad L^{\prime}\left(T, N_{\tau}\right) \equiv \frac{\partial L\left(T, N_{\tau}\right)}{\partial T} .
$$

Though the Polyakov loop in full QCD does not vanish below the crossover region, the presence of a peak in the entropy of a static quark is indicative of rapid change of the properties of the medium and the onset of deconfinement. The calculation of the peak position in $S_{Q}$ and the comparison of its value with the pseudo-critical temperature $T_{c}$ of the chiral crossover is important for improving our understanding of the chiral and deconfinement aspects of the finite temperature QCD crossover.

We calculate the temperature derivative of the free energy numerically. We use spline and polynomial interpolations using $\mathrm{R}$ statistical package [10] with error propagation via bootstrap method for the renormalization constant $c_{Q}(\beta)$ and for the bare free energy $f_{Q}^{\text {bare }}\left(\beta, N_{\tau}\right)$. We estimate the systematical error of our interpolation from the difference between both results. Further details of the interpolation are deferred to a publication in preparation [12]. Since our current data quality for $N_{\tau}=12$ is insufficient for precise calculation of the derivative, we show only results for $N_{\tau}=\{10,8,6\}$ in figure 2 .

The position of the peak agrees quite well with the pseudo-critical temperature $T_{c}$ obtained from chiral susceptibilities in [8]. We obtain $T_{\text {peak }}=159(3), 162(4)$ and $166(4) \mathrm{MeV}$ for $N_{\tau}=\{10,8,6\}$, which compares well with $T_{\mathrm{c}}=161.9(1.4), 164.1(1.8)$ and $171.3(2.1) \mathrm{MeV}[8]$. Below $T \lesssim 170 \mathrm{MeV}, N_{\tau}=6$ is not in the $1 / N_{\tau}^{2}$ scaling regime for continuum extrapolation of $S_{Q}\left(T, N_{\tau}\right)$. Hence, we cannot present a robust continuum extrapolation of the entropy for 
temperatures in the crossover region. Above $T \gtrsim 250 \mathrm{MeV}$, cutoff effects in $S_{Q}\left(T, N_{\tau}\right)$ are as large as the statistical uncertainties.

\section{Color screening observables from the static quark correlators}

Due to color screening, static quark-antiquark free energies approach the same constant value at sufficiently large distances. This constant equals to twice the free energy of an isolated static quark because the color charges are not correlated anymore. In other words the correlators defined above will approach $L^{2}$ at large distances. On the other hand, thermal effects are no more than a perturbation to correlators at sufficiently short distances. At distances $r \lesssim 1 / T$ we expect to see the interplay between vacuum physics and medium effects, while at still larger distance we should see the onset of color screening. It is convenient to subtract the known asymptotic constant and obtain correlators that vanish for sufficiently large distances. By subtracting the bare Polyakov loop squared from the correlation functions the correlated noise at large distances is greatly reduced. The renormalized correlators are obtained by adding back the asymptotic constant in terms of the renormalized instead of the bare Polyakov loops.

\subsection{Color-singlet free energy $F_{S}(r, T)$}

Applying the previously discussed approach to the color-singlet Wilson line correlator $C_{S}\left(r, T, N_{\tau}\right)$, we compute $f_{S}^{\text {sub }}\left(r, T, N_{\tau}\right)=f_{S}\left(r, \beta, N_{\tau}\right)-2 f_{Q}^{\text {bare }}\left(\beta, N_{\tau}\right)$, the subtracted color singlet free energy. As for the Poylakov loop, interpolation in the temperature is required for continuum extrapolation at fixed temperature. Moreover, the available lattice distances $r / a(\beta)$ correspond to different physical lengths for different $\beta$. We correct for leading cutoff effects of the tree-level gauge action at short distances by using the improved distance $r_{I}$ (see e.g. [13]) instead of bare distance $r_{b}$ and interpolate in the distance. We perform both pointwise continuum extrapolation for each temperature and distance and with a global fit using a polynomial Ansatz similar to eq. (7) in both the temperature and the distance. Finally we add the continuum limit of the renormalized asymptotic value, $2 f_{Q}^{\text {ren }}(T)$, and obtain the renormalized singlet free energy. The continuum limit of this result is shown together with the static energy at zero temperature, $V(r)$, in figure 3. Both quantities are numerically similar up to $r T \lesssim 0.45$. This indicates a regime of almost vacuum-like physics with medium effects suppressed for small $r T$. These two regimes can be made even more explicit by defining an effective coupling constant $\alpha_{Q \bar{Q}}(r, T)$ as

$$
\alpha_{Q \bar{Q}}\left(r, T, N_{\tau}\right)=-\frac{3}{4} r^{2} \frac{\partial E\left(r, T, N_{\tau}\right)}{\partial r}, \quad E\left(r, T, N_{\tau}\right)=\left\{F_{S}\left(r, T, N_{\tau}\right), V(r)\right\},
$$

which has only a mild $N_{\tau}$ dependence. We show $\alpha_{Q \bar{Q}}(r, T, 6)$ together with $\alpha_{Q \bar{Q}}(r, 0)$ obtained from the zero temperature static energy $V(r)$ in figure 5. The maximum of $\alpha_{Q \bar{Q}}\left(r, T, N_{\tau}\right)$ defines a distance $r_{\max }(T)$ at which screening overcomes the string tension. Thus, for $r<$ $r_{\max }(T)$ we see vacuum-like behavior, while for $r>r_{\max }(T)$ we see the onset of color screening.

\subsection{Color-averaged free energy $F_{\text {avg }}(r, T)$}

Applying the same machinery to the Polyakov loop correlator $C_{P}\left(r, T, N_{\tau}\right)$, we compute $f_{\text {avg }}^{\text {sub }}\left(r, T, N_{\tau}\right)=f_{\text {avg }}\left(r, \beta, N_{\tau}\right)-2 f_{Q}^{\text {bare }}\left(\beta, N_{\tau}\right)$, the subtracted color-averaged free energy. 


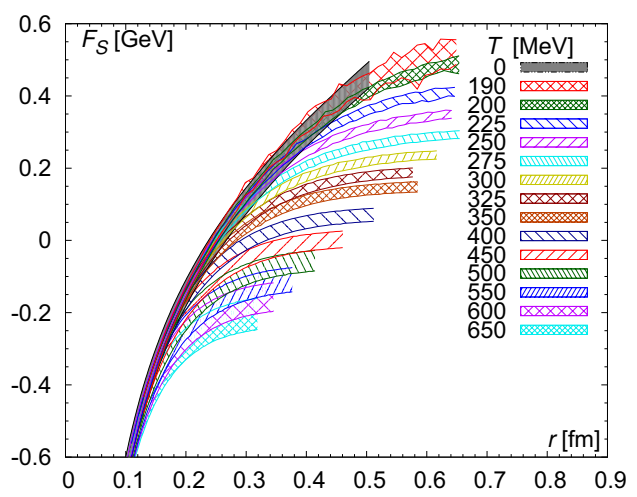

Figure 3. The singlet free energy $F_{S}(r, T)$ is numerically similar to the zero temperature static energy $V(r)$ up to $r T \lesssim 0.45$.

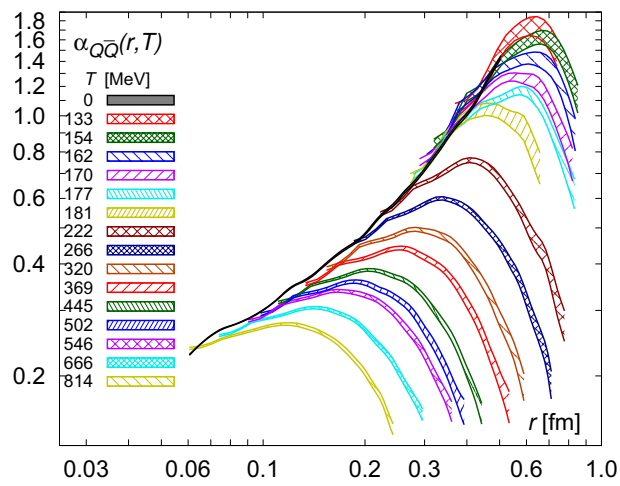

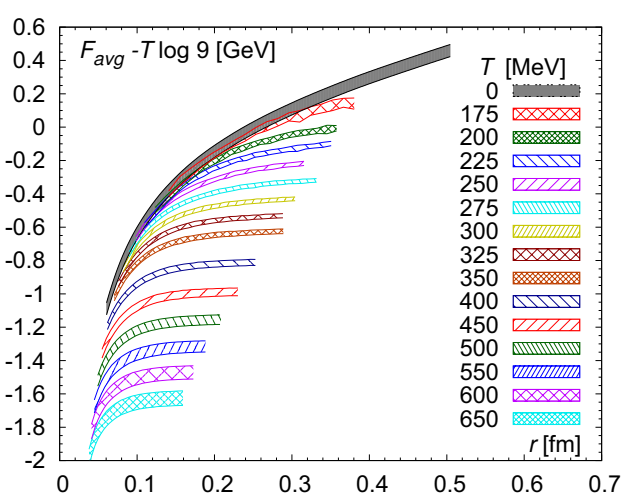

Figure 4. Once a trivial color factor $T \log 9$ is subtracted from the color-averaged free energy; $F_{\text {avg }}(r, T)$ it is numerically similar to the zero temperature static energy $V(r)$ up to $r T \lesssim 0.15$.

Figure 5. The effective coupling constant $\alpha_{Q \bar{Q}}(r, T)$ highlights the transition between confining and screening regimes (here $N_{\tau}=6$ ). The quadratic rise of the black band for $T=0$ is indicative of the string tension.

Due to combinatorics, it can be written in terms of the color-singlet free energy and the color-octet free energy ${ }^{1}$

$$
\exp \left(-f_{\mathrm{avg}}\left(r, \beta, N_{\tau}\right)\right)=\frac{1}{9} \exp \left(-f_{S}\left(r, \beta, N_{\tau}\right)\right)+\frac{8}{9} \exp \left(-f_{O}\left(r, \beta, N_{\tau}\right)\right)
$$

Hadronic states with $Q \bar{Q}$ pair in color-octet configuration require valence gluons and correspond to hybrid mesons, which are energetically disfavored at low temperatures. Furthermore, in octet channel we have repulsive interactions at short distances. Hence, it is expected that the color-averaged free energy is dominated by the singlet contribution for short enough distances: $F_{\text {avg }}\left(r, \beta, N_{\tau}\right) \simeq F_{S}\left(r, T, N_{\tau}\right)+T \log 9$. We account for the trivial color factor $T \log 9$ and show the modified continuum limit of the color-averaged free energy together with the static energy at zero temperature, $V(r)$, in figure 4. It is no surprise that differences between $F_{\text {avg }}(r, T)$ and $V(r)$ due to in-medium modification become significant for much smaller $r$ due to the rising contribution from states in color-octet configuration.

\footnotetext{
${ }^{1}$ The decomposition of the $Q \bar{Q}$ free energy into singlet and octet contributions can be rigorously derived in the small distance limit using effective field theory approach [14].
} 


\section{Conclusions}

We have studied the Polyakov loop, its correlator and a color-singlet Wilson line correlator in full QCD with $2+1$ flavors of quarks almost at the physical point. Using these observables, we calculate free energies and study deconfinement and color screening in the thermal medium.

We find that the Polyakov loop is a useful order parameter for deconfinement in full QCD. For low temperatures, renormalization using either $Q \bar{Q}$ procedure or gradient flow yields numerically consistent results up to cutoff effects. The deconfinement crossover is clearly indicated by a peak in the entropy $S_{Q}(T)$. The cutoff dependence of the associated temperature $T_{\text {peak }}$ and of the pseudo-critical temperature $T_{c}$ defined in terms of chiral observables are numerically consistent.

The free energies of static $Q \bar{Q}$ pairs in the medium are consistent with the zero temperature static energy $V(r)$ for short distances, indicating vacuum-like physics as required by asymptotic freedom. For larger distances, the normalized color-averaged free energy $F_{\text {avg }}-T \log 9$ starts to deviate from $V(r)$ at $r T \approx 0.15$ due to the presence of the octet contribution, whereas the color-singlet free energy $F_{S}(r, T)$ stays close to $V(r)$ up to $r T \approx 0.45$. We attribute this deviation to the onset of color screening and deconfinement, which is highlighted more explicitly in an effective coupling constant $\alpha_{Q \bar{Q}}(r, T)$.

\section{Acknowledgments}

This work was supported by U.S. Department of Energy under Contract No. DE-SC0012704. We acknowledge the support by the DFG Cluster of Excellence "Origin and Structure of the Universe". The calculations have been carried out on the computing facilities of the Computational Center for Particle and Astrophysics (C2PAP).

\section{References}

[1] L. D. McLerran and B. Svetitsky, Phys. Rev. D 24, 450 (1981).

[2] S. Mukherjee, P. Petreczky and S. Sharma, arXiv:1509.08887 [hep-lat].

[3] http://www.physics.utah.edu/ detar/milc/milc_qcd.html

[4] http://www.usqcd.org/software.html

[5] O. Kaczmarek, F. Karsch, P. Petreczky and F. Zantow, Phys. Lett. B 543, 41 (2002)

[6] S. Digal, S. Fortunato and P. Petreczky, Phys. Rev. D 68, 034008 (2003)

[7] A. Bazavov et al. [MILC Collaboration], PoS LATTICE 2010 (2010) 074

[8] A. Bazavov et al., Phys. Rev. D 85 (2012) 054503

[9] A. Bazavov et al. [HotQCD Collaboration], Phys. Rev. D 90 (2014) 9, 094503

[10] R Development Core Team (2011), R: A Language and Environment for Statistical Computing. Vienna, Austria : the R Foundation for Statistical Computing. ISBN: 3-900051-070, http://www.R-project.org/.

[11] P. Petreczky and H.-P. Schadler, Phys. Rev. D 92 (2015) 9, 094517

[12] Preprint: A. Bazavov, N. Brambilla, H.T. Ding, P. Petrczky, H.-P. Schadler, A. Vairo, J. Weber, Polyakov loop in $2+1$ flavor QCD from low to high temperatures, TUM-EFT $76 / 15$

[13] A. Bazavov, N. Brambilla, X. Garcia i Tormo, P. Petreczky, J. Soto and A. Vairo, Phys. Rev. D 90, 074038 (2014)

[14] N. Brambilla, J. Ghiglieri, P. Petreczky and A. Vairo, Phys. Rev. D 82, 074019 (2010) 